\title{
Does symmetry structure facilitate the depth separation between stereoscopically overlapped dot planes?
}

\author{
AKIRA ISHIGUCHI and REIKO YAKUSHIJIN \\ Ochanomizu University, Tokyo, Japan
}

\begin{abstract}
In the present study, we dealt with the problem of whether a symmetrical structure can influence the discrimination of the depth separation of overlapped dot planes. We investigated this problem with the use of both direct and indirect methods. In the direct method, we presented three or two overlapped dot planes consisting of symmetrical or random dots. The subjects were required to discriminate three overlapped from two overlapped planes. In the indirect method, the subjects were required to discriminate the depth positions of a target dot (or a pair of dots) that disappeared during stimulus presentation. Our results, obtained in three experiments, showed that the discrimination performance improved and reached a perfect level in the direct method and a modest plateau level in the indirect method with increasing relative disparity between the two outer planes, irrespective of whether the dot pattern had a symmetrical structure or not. These results suggest that a detection process for symmetry structure on a two-dimensional plane in three-dimensional space will not have a direct or an indirect connection (e.g., via a feedback loop) to a process involved in the depth separation.
\end{abstract}

Random dots with an equal disparity value produce a percept of a flat surface in a three dimensional (3-D) space. On the other hand, when dots with more than one disparity value are presented within a region, some different perceptual interpretations occur, depending on the magnitude of these disparity values. Recent work suggests that one of the following three percepts occurs when one observes a random dot pattern in which two distinctly different disparity values are present within the same spatial region (Parker \& Yang, 1989; Stevenson, Cormack, \& Schor, 1989). First, when the relative disparity between two groups of components is small enough, a single flat surface, which is sometimes perturbed by noise, is perceived. Second, when the relative disparity is large enough, two overlapped surfaces and a vacant space between them are perceived. In this case, the front surface is perceived to be transparent. This percept implies that elements with the same disparity values are grouped to construct the two overlapped surfaces. Third, when the relative disparity lies between the first and the second cases, a single thick board is perceived, but some elements appear to float within the space between the front and back surfaces of the board. The third intermediate state is termed pykno stereopsis by Tyler (1983).

In regard to the above second case, Stevenson et al. (1989) measured the threshold for depth separation of two overlapped dot planes and termed it gap resolution acu-

This research was supported by a Grant-in-Aid for Scientific Research (C) No. 07610069 to A. I. from the Ministry of Education, Science and Culture in Japan. We are grateful to Mike Braunstein and anonymous reviewers for their valuable comments. Correspondence concerning this article should be addressed to A. Ishiguchi, Department of Human and Social Sciences, Ochanomizu University, 2-1-1 Otsuka, Bunkyo-ku, Tokyo 112, Japan (e-mail: akiral@li.ocha.ac.jp). ity. According to their results, the threshold, which depends on subjects, was from 2 to, at most, 6 min of arc.

The gap resolution acuity is defined by a threshold value for a task that tests whether two overlapped planes appear to be separated in depth. In this situation, we are much interested in the problem of whether structured dot planes have some facilitative effects on the depth separation. Probably, two processes are involved in the depth separation of overlapped dot planes. One is a grouping process in which dot elements are divided into groups on the basis of an internal representation of their binocular disparities. The other is an averaging process in which some sample disparities drawn from dots in a group are averaged to produce a smooth surface. Along this line, we assumed two possible ways in which the facilitative effects might occur. One possibility is that the detection of spatial structures will promote the grouping of pattern elements. The other possibility is related to a statistical sampling theory: A large sample reduces the variance of the sample mean. If detection of spatial structures increases the sample size of pattern elements in the averaging process, the variance of the internal distribution of their disparity values will be reduced (see the Discussion section for Experiment 3). These effects can occur via a feedback output from a higher stage, even if the detection process for the structure lies in the higher stage of visual processing. Nakayama, Shimojo, and Ramachandran (1990) suggested some feedback effects from a higher stage on more primitive processes.

In this study, we dealt with symmetry structure, because this spatial structure is omnipresent in our daily life. Thus, the problem is whether a smaller relative disparity is needed to separate stereoscopically overlapped dot planes when the dots are arrayed symmetrically (see Figure 1). 
Yakushijin and Ishiguchi (1999) investigated one aspect of the relation between the symmetry structure and the depth separation of overlapped planes. They presented sequentially a couple of 3-D stimuli, each of which consisted of two stereoscopically overlapped dot planesa symmetrical dot pattern ( $100 \%$ or $50 \%$ symmetry) in one depth plane and a random dot pattern $(0 \%$ symmetry) in the other - and examined the effect of elements in the latter plane (i.e., the noise plane) on the discrimination of symmetry in the former plane (i.e., $100 \%$ vs. $50 \%$ in the target plane). Their results showed that the discrimination was improved with relative disparity between the overlapped planes and that the effect of the noise plane disappeared, although not completely, when the relative disparity was greater than $4 \mathrm{~min}$ of arc. In this case, they assumed that the discrimination performance would reach that of $100 \%$ versus $50 \%$ symmetry discrimination in two-dimensional (2-D) space without noise when the overlapped depth planes were separated completely. Their main concern was to study the effect of depth separation on the symmetry discrimination, not to study the effect of symmetry structure on the depth separation. We investigated the latter problem in this research.

The purpose of the present study was to examine the possibility that a symmetry structure facilitates the depth separation of stereoscopically overlapped dot planes. In order to do this, we used two methods: a direct and global discrimination method in Experiment 1 and an indirect and local discrimination method in Experiments 2 and 3. In the direct method, we presented two or three overlapped dot planes consisting of symmetrically arranged dots or random dots. The subjects were required to discriminate between three overlapped and two overlapped dot planes. This task resembles that for the measurement of the gap resolution acuity. Next, in Experiments 2 and 3 , we investigated the same problem with the use of an indirect and local discrimination task in which the subjects were required to choose the plane from which some of the dot elements were removed in the latter part of the stimulus presentation time. We compared percent correct data in the symmetry dot planes condition with those in the random dot planes condition. We assumed that the indirect and local discrimination method was necessary to examine the above problem. This is necessary because some floating dots are perceived to float within a space between two overlapped planes in depth when the relative disparity between them is not so large, and one must be able to choose the plane to which an arbitrary stimulus dot belongs when two overlapped planes are perceived clearly to be separated.

We could predict some parts of our results when using these methods. With the global method, we will not need to discriminate between falsely grouped dots and truly grouped ones. However, with the local method, we will be faced with the problem of whether an arbitrary dot is grouped truly. Thus, a more distinct depth separation will be needed in the latter case, and the relative disparity for the depth separation may be larger when using the local method than when using the global method.

It should be noted that, although we surmise there to be a relation between these two methods and the above two processes (i.e., the grouping and averaging processes), we did not intend to clarify the two processes by using these two methods in this study. We intended to clarify the relation between the symmetrical structure and the depth separation of the overlapped depth planes in the following experiments.

\section{EXPERIMENT 1}

\section{Method}

Subjects. Four subjects-the authors, a graduate student, and an undergraduate student-participated in the following experiments. The two authors were much experienced in the depth separation experiments. The purpose of the experiments was unknown to the other subjects. All the subjects had normal or corrected-to-normal acuity and good stereopsis.

Apparatus. The stimuli were generated by a Macintosh Quadra 800 and presented on a pair of 17 -in. color CRT displays
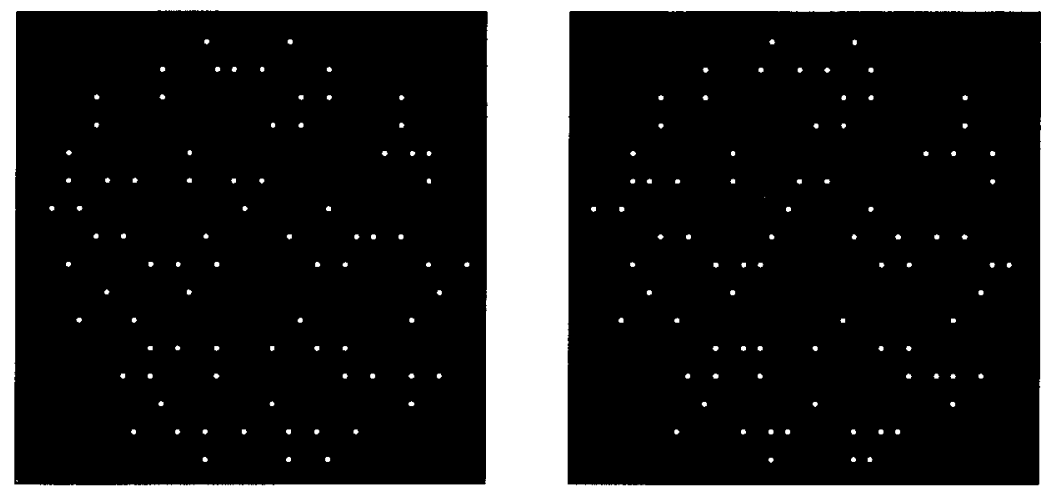

Figure 1. This pair of dot patterns, if fused, produces a symmetrical dot surface (e.g., a surface where mirror-symmetrical dots about a vertical axis are scattered) overlapped by a random dot surface. The front random dot surface appears transparent. 
(TOTOKU CV173). The subjects viewed the stimuli through a stereoscope consisting of two pairs of surface mirrors and the pair of monitors from a distance of $232 \mathrm{~cm}$. This stereoscope device was adjusted so that the two eyes could converge at this distance. We used a chin rest to stabilize loosely the head position.

Stimuli. Displays consisted of 144 bright dots $\left(29 \mathrm{~cd} / \mathrm{m}^{2}\right)$ on a dark background $\left(0.3 \mathrm{~cd} / \mathrm{m}^{2}\right)$, Each dot subtended about $1 \times 1 \mathrm{~min}$ of arc. The stimulus dots were separated from each other by at least $13 \mathrm{~min}$ of arc in a fused image. A whole display was presented in a squared area of $3.14 \times 3.14 \mathrm{deg}$ of arc. The stimulus dots were arrayed in two or three stereoscopically overlapped planes. In the pattern of two overlapped planes, 72 dots were arrayed in each plane; in the pattern of three overlapped planes, 48 dots were presented in each plane. Thus, in both patterns, the same number of dots (i.e., 144 dots) was presented as a whole. The arrangement of dots in each plane was either symmetry about a vertical axis or nonsymmetry ( $0 \%$ bilateral symmetry, termed random henceforth).

Design and Task. Each stimulus pattern consisted of two or three stereoscopically overlapped planes. The subjects were required to make two observations and then to choose the observation interval that contained the pattern consisting of three overlapped planes.

We made two structure conditions: symmetry and random. In the symmetry condition, the three-plane patterns consisted of two $100 \%$ symmetry dot planes and a random dot plane (i.e., $0 \%$ symmetry) that was interlaid between the symmetry planes, and the two-plane patterns consisted of two $67 \%$ symmetry planes. We manipulated both three-plane and two-plane patterns to yield the same degree of symmetry pattern in the frontoparallel plane (i.e., $67 \%$ symmetry). Thus, the subject could not use 2-D symmetry cues in the projection plane in discriminating between three overlapped and two overlapped dot planes. In the random condition, both the three-plane and the two-plane patterns consisted of $0 \%$ symmetry dot planes and, at the same time, yielded $0 \%$ symmetry pattern in the projection plane. Figure 2 will make clear the above experimental design.

We combined the structure conditions with four relative binocular disparities between the outer planes in both the two- and the three-plane patterns. Four values of the relative disparity, depending on subjects (i.e., 1, 2, 3, and $4 \mathrm{~min}$ for A.I., R.Y., and M.I.; 2, 4, 6, and $8 \mathrm{~min}$ for M.H.), were examined. In the three-plane pattern, we presented the other dot plane in the middle depth of the outer dot planes. In a single experimental block, eight combinations of these conditions (two structure conditions $\times$ four disparity conditions) were each presented 10 times in a random order. Twenty experimental blocks were run for each subject.

Procedure. A two-alternative forced-choice (2AFC) method with two successive stimulus intervals was used. A trial was initiated by a subject's keypress, and a fixation point was presented for $500 \mathrm{msec}$. The depth position of the fixation point was in the middle of the outer stimulus dot planes. Then, a 500-msec blank interval and a couple of stimulus observation intervals followed. Each observation phase lasted $1.0 \mathrm{sec}$, and an interstimulus interval had a duration of $500 \mathrm{msec}$. The subjects were required to make two observations and then to choose the observation phase (i.e., a target phase) that contained the three-plane pattern. The subjects responded by pressing one of two keys indicating the target phase. Feedback was provided for incorrect responses by a beep sound. The next trial was started by the subjects' making another keypress after their response. Each subject did 16 practice trials before each experimental block.

\section{Results and Discussion}

Percent corrects are based on a nonlinear axis metric. Thus, to stabilize variances and obtain maximal statistical power (Winer, 1962), we applied the arcsine transformation to the percent correct data for the 2AFC discrimination tasks. The following statistical analyses were made on the transformed data.

\section{SYMMETRY Condition}
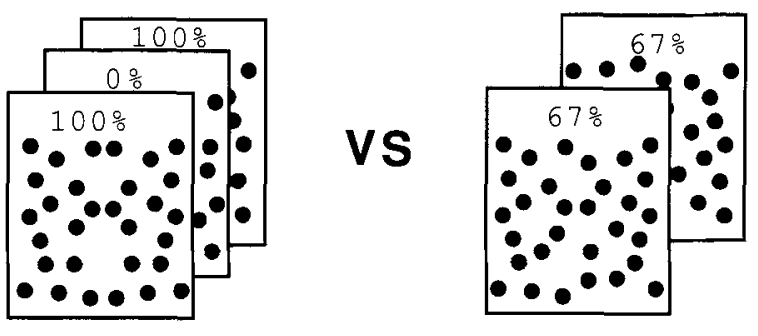

\section{RANDOM Condition}
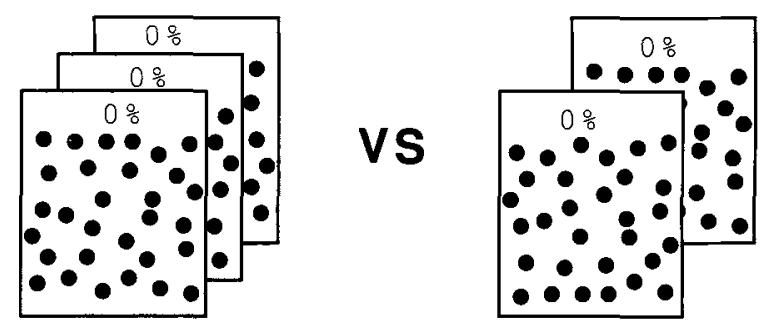

Figure 2. A schematic representation of the structure conditions. Each sheet indicates a dot plane overlapped in depth. Numerical values in the sheet indicate the degree of symmetry .

Figure 3 shows the percent correct for each subject as a function of the relative disparity between the two outer planes. Closed squares and open circles indicate results for the random condition and for the symmetry condition, respectively.

As Figure 3 shows, the discrimination of patterns appears to improve steeply with the relative disparity of up to about 3 (A.I., R.Y., and M.I.) or $6 \mathrm{~min}$ (M.H.), then to reach almost the perfect level. It is obvious that this tendency is not affected by the structure conditions (i.e., symmetry vs. random).

The statistical analyses supported these tendencies. A two-way repeated measures analysis of variance (ANOVA) conducted on each subject's data set was used to examine both the effects of the structure conditions and those of the relative disparity. Twenty experimental blocks were used as the repetition measurement. The effects of structure conditions were not significant, except for the results for 1 subject, M.I. $[F(1,19)=6.08, p<.05]$. The results for the other 3 subjects revealed no difference between the symmetry and the random conditions. On the other hand, the ANOVA revealed a significant difference among relative disparities [A.I., $F(3,57)=216.2, p<.0001$; R.Y., $F(3,57)=92.36, p<.0001 ;$ M.I., $F(3,57)=171.3, p<$ $.0001 ;$ M.H., $F(3,57)=163.3, p<.0001]$. 

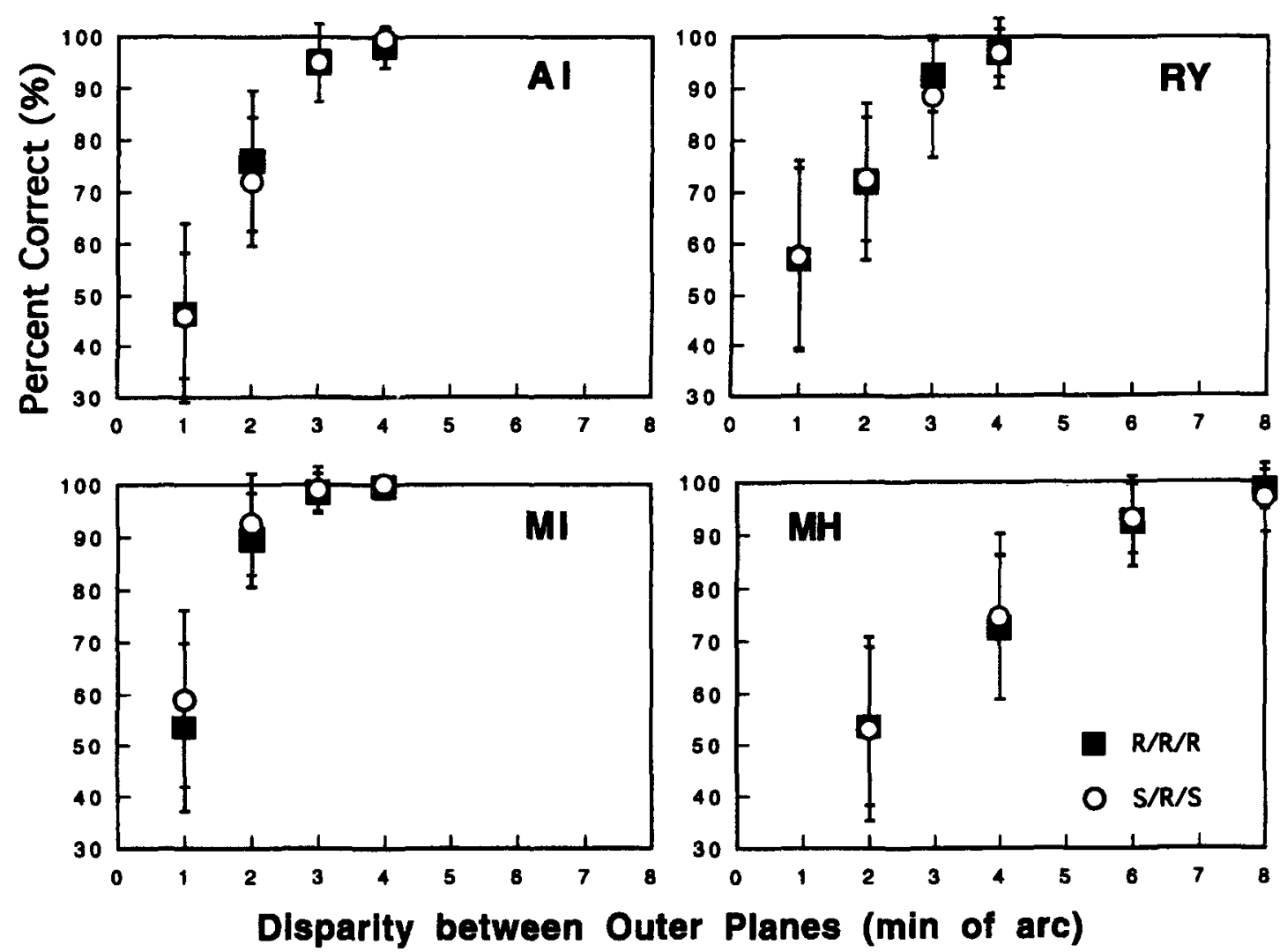

Figure 3. The results in Experiment 1: percent correct for each subject as a function of the relative disparity between the two outer dot planes. Closed squares and open circles indicate results for the random condition and for the symmetry condition, respectively. Vertical error bars represent standard deviations.

Thus, we can conclude that the direct and global method revealed no clear effects of the symmetry structure on the depth separation of stereoscopically overlapped planes. The results in this experiment showed that $78 \%$ percent correct was obtained when the relative disparity between the outer planes was from about 2-4 min of arc. Although we were not interested directly in the threshold for the depth separation, these data were almost consistent with the gap resolution acuity measured by Stevenson et al. (1989).

In Experiment 2, we used an indirect and local discrimination method to investigate the influence of dot structure on the depth separation of two overlapped dot planes. The indirect method was to use a discrimination task in which the subjects were required to choose the plane from which a single dot was removed in the latter end of stimulus presentation time. We used only two dot planes that were stereoscopically overlapped. The dot planes had a symmetry or a random structure.

\section{EXPERIMENT 2}

\section{Method}

Subjects and Apparatus. Both subjects and apparatus were the same as those in Experiment 1.

Stimuli. Displays consisted of bright dots $\left(29 \mathrm{~cd} / \mathrm{m}^{2}\right)$ on a dark background $\left(0.3 \mathrm{~cd} / \mathrm{m}^{2}\right)$. Each dot subtended about $1 \times 1 \mathrm{~min}$ of arc. They were separated from each other by at least $13 \mathrm{~min}$ of arc in a fused image. A stimulus pattern was composed of two dot planes that had different disparity values. Each plane contained 40 dots. This manipulation produced a percept of a pair of dot surfaces overlapped in depth. The arrangement of dots in each dot group was either symmetry about a vertical axis or nonsymmetry $(0 \%$ symmetry, termed random, as mentioned before).

A single dot (i.e., a target) contained in one of the dot planes disappeared $1.0 \mathrm{sec}$ after onset of the stimulus pattern. This target dot was chosen randomly from dots within a central circular region occupying a quarter of the whole display area. A whole display was presented in a circular area with 3.14 deg of arc in diameter.

Design and Tasks. Three kinds of 3-D dot patterns, each of which had two overlapped dot planes, were displayed randomly in a single experimental block: symmetry versus symmetry dot planes pattern, symmetry versus random dot planes pattern, and random versus random dot planes pattern. We termed them the structure conditions. The symmetry pattern was composed of dots arranged to be mirror symmetry about a vertical axis. The random pattern was composed of dots arranged to be $0 \%$ symmetry about the axis. The relative disparities between the two overlapped dot planes were varied $(2,4,8$, and $16 \mathrm{~min}$ of arc).

In each experimental block, 12 combinations of these conditions (three structure conditions $\times$ four relative disparities) were each presented 20 times in random order. In half of the trials of each block, the target was chosen from dots in the front plane, and in another half, it was chosen from dots in the back plane. The depth positions of the target were randomly alternated. The subjects were required to choose the plane from which a single dot disappeared in the lat- 
ter part of the stimulus presentation time. Ten experimental blocks were run for each subject.

Procedure. A spatial 2AFC method with one stimulus interval was used. A trial was initiated by the subject's keypress. A 500-msec blank time was followed by a stimulus presentation. A stimulus pattern (i.e., two overlapped dot planes) was presented for $1.5 \mathrm{sec}$. A single target dot disappeared $1.0 \mathrm{sec}$ after the stimulus onset. The subject's task was to discriminate the depth positions of the plane from which the target disappeared. The subject responded by one of two keys indicating the depth positions of the target. Feedback was provided for incorrect responses by a beep sound. The next trial was started by the subjects' making another keypress after their responses. Each subject did 24 practice trials before each experimental block.

\section{Results and Discussion}

We applied the arcsine transformation to percent correct data for $2 \mathrm{AFC}$ discrimination tasks, and the following statistical analyses were made on the transformed data.

At first, because the effect of the target depth position was negligible, the data for it were pooled.

Figure 4 shows the mean percent correct for the 4 subjects as a function of the relative disparity between the two overlapped dot planes. The averaged data were used because the disparity values examined (i.e., 2, 4, 8, and $16 \mathrm{~min}$ of arc) were common to the 4 subjects. Open circles, half-closed squares, and closed squares indicate results for the symmetry versus symmetry, the symmetry versus random, and the random versus random pattern conditions, respectively.

As Figure 4 shows, discriminability for the target depth position appears to improve gradually with the relative disparity up to about $8 \mathrm{~min}$, then to reach a plateau of modest magnitude, irrespective of the structure conditions. The statistical analyses supported these tendencies. A

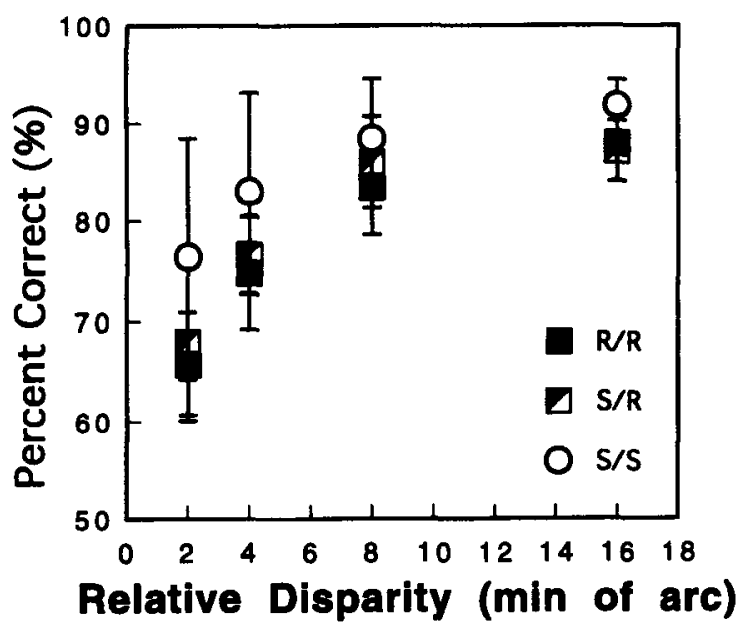

Figure 4. The results in Experiment 2: mean percent correct across the 4 subjects as a function of the relative disparity between two overlapped dot planes. Open circles, half-closed squares, and closed squares indicate results for the symmetry versus symmetry pattern, for the symmetry versus random pattern, and for the random versus random pattern conditions, respectively. Vertical error bars represent standard errors. two-way repeated measures ANOVA conducted on the full data set was used to examine both the effects of the relative disparity and those of the structure conditions. The effect of the relative disparities was significant $[F(3,9)=26.96, p<.001]$. Application of Tukey method showed that there were significant differences between the following pairs of disparity conditions- 2 versus $4 \mathrm{~min}$, 2 versus $8 \mathrm{~min}, 2$ versus $16 \mathrm{~min}, 4$ versus $8 \mathrm{~min}$, and 4 versus $16 \min (p<.01)$ - but not significant between 8 versus 16 min conditions.

There were more important results for the structure condition. The ANOVA revealed a significant difference among three kinds of structure conditions $[F(2,6)=$ $8.28, p<.5]$. Application of the Tukey method showed a better performance in the symmetry versus symmetry condition than in the other conditions $(p<.05)$.

It is possible that the better discrimination obtained in the symmetry versus symmetry condition in this experiment was due to an auxiliary clue to the symmetry pattern. Because the mate to the target dot (i.e., one of the mates of a symmetrical pair) remained visible for $500 \mathrm{msec}$ in the symmetry versus symmetry condition after the target dot had disappeared, the subjects could decide which plane the target had belonged to by using the perceived depth of the mate as a clue. It is certain that we could also use the mate's depth clue in the symmetry versus random condition. However, perception of symmetry was enhanced only in the symmetry versus symmetry condition, because the stimulus pattern in this condition was still perfectly symmetrical in the projection plane (i.e., in the frontoparallel plane). On the other hand, in the symmetry versus random condition, the pattern was asymmetrical in the projection plane. Thus, the discrimination in the symmetry versus random condition was not so good as that in the symmetry versus symmetry condition.

In Experiment 3, we used, as a target, a pair of dots instead of a single dot and removed the auxiliary clue in the symmetry versus symmetry condition.

\section{EXPERIMENT 3}

\section{Method}

The method in this experiment was the same as that in Experiment 2, except that a pair of dots, instead of a single dot, was used as a target. In addition, the symmetry versus random pattern condition was excluded. This is because the results for the symmetry versus random condition were not different from those for the random versus random condition in Experiment 2, and because an arrangement of a target dot pair in Experiment 3 may be used by the subjects as a clue in discriminating between the overlapped symmetry and random dot planes in the symmetry versus random condition. That is, if a target pair is found to be symmetrical, one can choose a symmetrical dot plane, and if a target pair is found not to be symmetrical, one can choose a random dot plane. In these situations, the subjects will judge the depth separation in a global, not a local, manner, because their judgment could be based on the depth position of the global pattern. Two other conditions (i.e., the symmetry vs. symmetry and the random vs. random conditions) were examined.

In the symmetry versus symmetry condition, the target pair was chosen from symmetrical pairs. In the random versus random con- 
dition, the target pair was chosen from dots aligned horizontally, one dot from the left half and another dot from the right half of the random plane. This manipulation made the target pair to be aligned in the same orientation under both conditions.

Procedure. A spatial 2AFC method with one stimulus interval was used. A single trial proceeded in the same way as that in Experiment 2. A stimulus pattern (i.e., two overlapped dot planes) was presented for $1.5 \mathrm{sec}$, and a pair of target dots disappeared $1.0 \mathrm{sec}$ after the stimulus onset. The subjects' task was to discriminate the depth positions of the target.

\section{Results and Discussion}

We applied the arcsine transformation to the percent correct data for $2 \mathrm{AFC}$ discrimination tasks, and the following statistical analyses were made on the transformed data.

Figure 5 shows the mean percent correct for the 4 subjects as a function of relative disparity between two overlapped dot planes. Open circles and closed squares indicate the results for the symmetry versus symmetry condition and for the random versus random condition, respectively.

As is shown in Figure 5, the results in this experiment reconfirmed the tendency that the percent correct for the discrimination task gradually improved and reached a plateau level of a modest magnitude as the relative disparity was increased. However, the structure conditions in this experiment appeared to have little effect on the discrimination task. The statistical analyses supported these tendencies. A repeated measures ANOVA conducted on the full data set was used to test both the effects of the relative disparity and those of the structure conditions. The effects of the relative disparity were significant $[F(3,9)=$

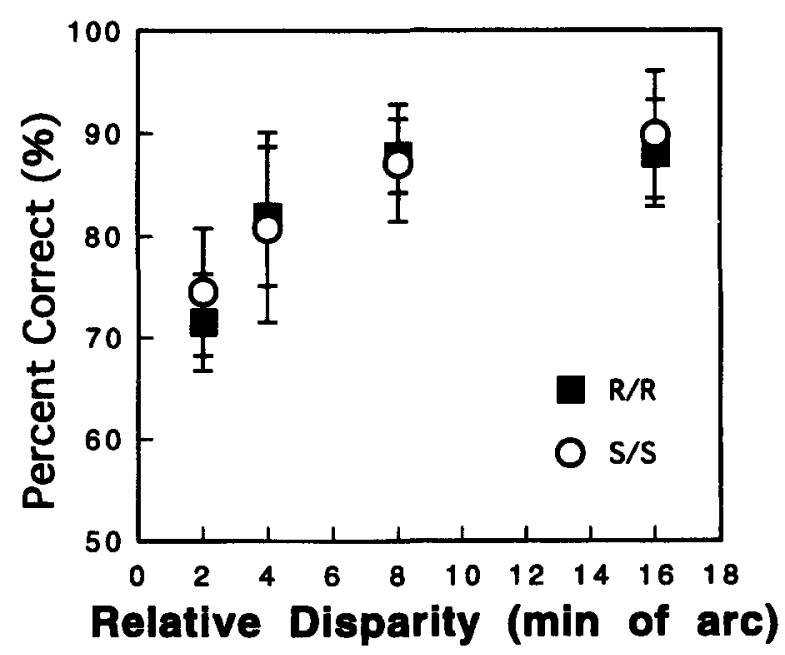

Figure 5. The results in Experiment 3: mean percent correct across the 4 subjects as a function of the relative disparity between two overlapped dot planes. Open circles and closed squares indicate results for the symmetry versus symmetry condition and for the random versus random condition, respectively. Vertical error bars represent standard errors.
$27.09, p<.0001]$. Application of the Tukey method showed that there were significant differences between the following pairs of conditions-2 versus $4 \mathrm{~min}, 2$ versus $8 \mathrm{~min}, 2$ versus $16 \mathrm{~min}, 4$ versus $8 \mathrm{~min}$, and 4 versus $16 \min (p<.01)$ - but not between 8 versus $16 \mathrm{~min}$ conditions.

More important is that the difference between the symmetry versus symmetry and the random versus random conditions was not significant $[F(1.3)=2.447, p=.216]$. In Experiment 1, the data for one subject (M.I.) showed a significant effect of the structure conditions on the depth separation. Thus, as in Experiment 1, a two-way repeated measures ANOVA conducted on each subject's data set was used to examine both the effects of the structure conditions and those of the relative disparity. Ten experimental blocks were used as the repetition measurement. For all the subjects, the effects of structure conditions were not significant [e.g., M.I.: $F(1,9)=0.122, p=.74$ ]

Because we excluded auxiliary clues for depth separation from the symmetry versus symmetry condition, we obtained more confident results about whether the symmetry structure had any effects on the depth separation of overlapped planes. From the above results, we can conclude that the symmetry structure has no facilitative effects on the depth separation of overlapped dot planes.

\section{INTERNAL GAUSSIAN FUNCTION MODEL AND A MASKING EFFECT}

We then had to consider the relation of our results to the gap resolution acuity and its model (Stevenson et al., 1989). In order to understand the significance of the gap resolution acuity, we had to consider the internal representation of the binocular disparity. If physical binocular disparity values are represented correctly in the visual system, detection probability of a depth-axis gap between two overlapped planes can be described as a step function of the relative disparity. However, in practice, the probability varies continuously and is described as a normal probability distribution function (i.e., the Gaussian function), or the Gaussian function as approximation. This is partly because optical properties in the eye and intrinsic noise in the visual system make the disparity value fluctuate, as has been described by classical psychophysics (Gescheider, 1985).

It is possible, in addition, that a filtering mechanism (e.g., the Gaussian filter) operates on the input in the visual system. If the left and right retinal images of randomdot patterns are filtered out along the visual pathway and the filtering is performed with the Gaussian function, the cross-correlation between the filtered images produces the Gaussian function, which has a larger standard deviation by $\sqrt{2}$ than the original Gaussian filter. This is because the cross-correlation function between the left and right portion of the random-dot stereogram is a unit impulse function, and the cross-correlation between the filtered images of the stereogram is the unit impulse func- 
tion convolved with the shape of the filter itself (Parker, Johnston, Mansfield, \& Yang, 1991).

Thus, in both cases, two internal Gaussian functions will be obtained in the visual system when two randomdot planes are stereoscopically overlapped. Here, we reconsider the aforementioned two processes that will be involved in the depth separation of overlapped dot planes. In the grouping process, the element dots will be divided into two groups on the basis of the internal disparity values, of which the probability will be described by the Gaussian function. In addition, we should pay attention to the fact that random dots of equal disparity value produce a percept of a flat surface in a 3-D space. If the internal representation of the disparity value fluctuates as a random variable, some smoothing or averaging mechanisms will be needed to produce the flat surface. Parker and Yang (1989) showed that two different depth planes were seen as a single surface at the average of two disparities under some conditions. We assume that this kind of averaging mechanism operates on the internal represen- tation of binocular disparities. When the relative disparity between two depth planes is too small to divide the dots into different groups, the averaging mechanism produces a single flat surface. Because the operation of the averaging mechanism is restricted by the magnitude of the relative disparity (Parker \& Yang, 1989), a multiple global averaging will work independently and produce two or more overlapped surfaces under a large relative disparity. For simplicity, we assume a situation in which two dot planes were overlapped in depth. In the averaging process, some samples of the disparity values drawn from each of two groups will be averaged to produce a couple of flat surfaces. The probability of the disparity value of each flat surface, which is the same as the averaged disparity across the sampled dots, will be described as the Gaussian function with smaller variance. Thus, in both processes, it is assumed that the depth separation is related to the degree of the separation of the two internal Gaussian functions (see Figure 6) and that the difference of means $(\Delta d)$ and the standard deviations $\left(\sigma_{1}, \sigma_{2}\right)$ of the functions will
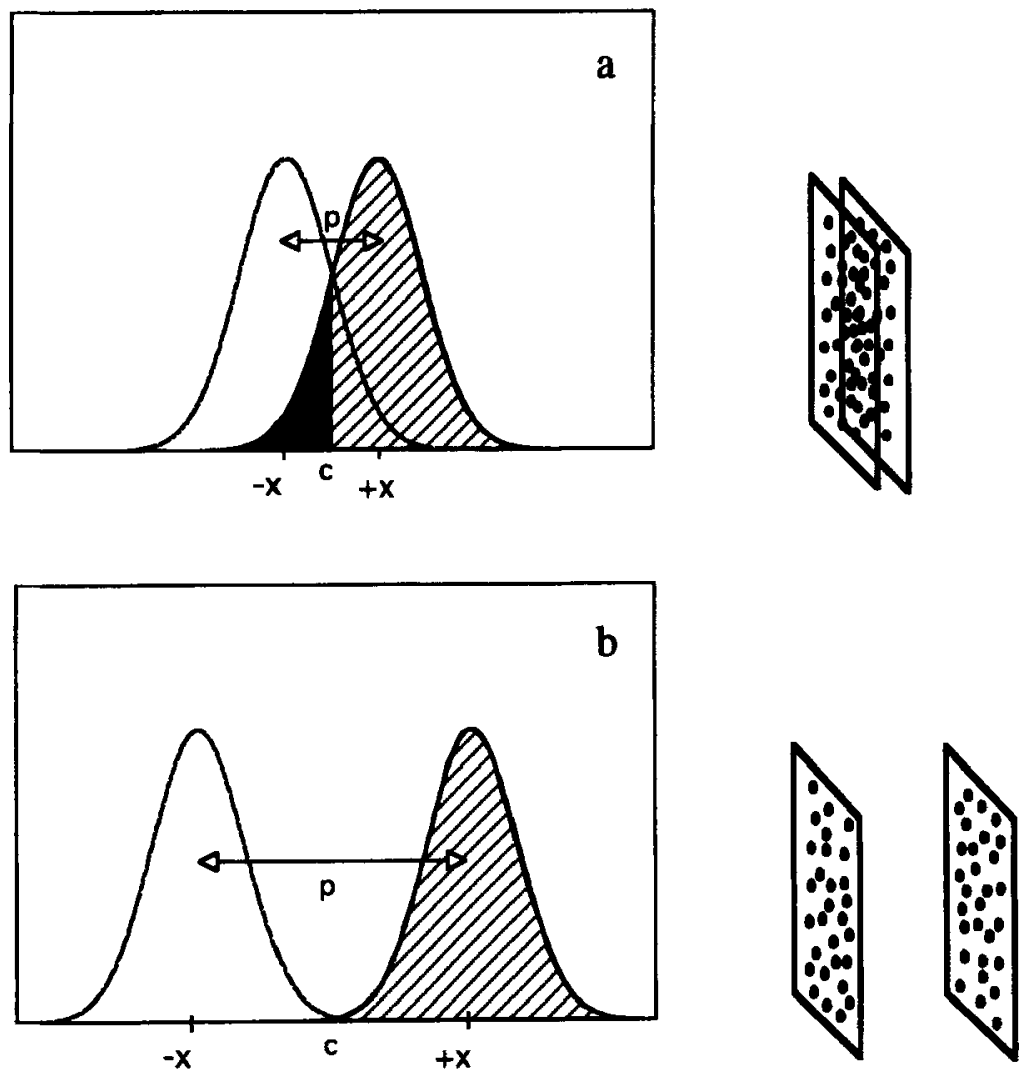

Figure 6. Schematic diagram of two overlapped probability density functions for the internal representation of binocular disparities. The two signed $\bar{x} s$ indicate physical disparity values, and $p$ is the probability that a dot image of one surface can interact with that of another surface. The relative disparity between two planes is small (panel a) and large (panel b). The gray area in panel a represents a probability of falsely grouped dots. The right column of this panel depicts a perspective view of the two overlapped surfaces. 
determine the perceived depth separation. However, we have dealt with these two processes as a whole up to now, because we could not independently investigate each of these two processes.

We assumed that, if detection of the symmetry structure has a facilitative effect on the depth separation, the effect will occur through reduction of the standard deviation of the Gaussian function for the symmetry dot pattern: The symmetry structure may reduce directly the fluctuation of the internal disparity in the grouping process, or it may reduce the standard error by increasing the size of the sample in the averaging process. Considering our result that there were no clear facilitative effects of symmetry structure on the depth separation of overlapped dot planes, the standard deviation (or standard error) of the assumed internal Gaussian function for the symmetry pattern will be the same as that for the random pattern. At any rate, it seems that the process of extracting a structure in a plane has no interaction with the process of extracting depth information and of constructing a surface.

We found, however, that only the internal Gaussian function model could not explain all the results in our experiments: The problem is that discrimination performance reached a modest plateau as the relative disparity increased, in Experiments 2 and 3. According to the internal Gaussian function model, percent correct improves with the relative disparity and reaches about the $100 \%$ level, not a modest plateau. This is because two internal Gaussian functions must have almost no overlaps when the relative disparity increases sufficiently. We will bring forward a supplementary model to settle this problem.

When two dot planes are overlapped in depth, we should pay attention to one dot plane to perform efficiently a task such as ours in Experiment 2 and 3. This strategy seems good because, if we can detect a missing target in the plane to which we pay attention, we can estimate the target depth directly. Even if we cannot detect the target in the plane, we can estimate the target depth indirectly, because the target had lain in the other plane, to which we do not pay attention. However, this strategy produces the inevitable result that an off-attended image is projected on the onattended image and plays a role of a mask. We are not concerned with the problem of whether these masking effects are memory- or perception-based, but we believe that this masking effect will be specific to the results in Experiments 2 and 3. In Experiment 1, our subjects had no need to pay attention to one surface to perform their task, and the masking effect was negligible. Thus, we supposed that at least two factors determined the discrimination performance in our Experiments 2 and 3: the interal probability functions for the binocular disparity and the masking effect. Furthermore, we assumed that the masking effect was constant within some range of the magnitude of the relative disparity between two overlapped dot planes. This assumption was inferred from our result that the discrimination performance reached a modest plateau. On the basis of these considerations, we formulated the percent correct (PC) in our tasks as the following equation, which will hold irrespective of the two processes above:

$$
\begin{aligned}
\mathrm{PC} & =p_{\text {mask }} \int_{-\infty}^{c} f(x) d x+\left(1-p_{\text {mask }}\right) \int_{c}^{+\infty} f(x) d x \\
f(x) & =\frac{1}{\sqrt{2 \pi} \sigma} e^{\frac{-1}{2}\left(\frac{x-\bar{x}}{\sigma}\right)^{2}},
\end{aligned}
$$

where $c$ indicates a critical disparity value at which two overlapped probability density functions ( $p d f s$ ) should be separated, and $\bar{x}$ and $\sigma$ indicate the mean and standard deviation (or standard error) of one of the $p d f s$ of the internal disparity distribution. The $\sigma$ involved in the averaging process will be smaller than that in the grouping process. The $c$ is considered to be an average for respective means of two disparity $p d f \mathrm{~s}$. The $p_{\text {mask }}$ indicates the probability that the dot images in one surface will interact with those in another surface (i.e., the masking effect). The $p_{\text {mask }}$ was used only to explain the results in Experiments 2 and 3 . We applied this model to our data. The disparity $p d f$ s were assumed to be $N\left(+\bar{x}, \sigma^{2}\right)$ and $N(-\bar{x}$, $\left.\sigma^{2}\right)$ where $\pm \bar{x}$ indicates physical disparity values. The standard deviation $(\sigma)$ and the probability $\left(p_{\text {mask }}\right)$ reflecting the masking effect were estimated by using the method of the least square error.

The estimated standard deviations of assumed internal Gaussian functions in Experiment 3 were as follows: 2.0 (A.I.), 1.9 (R.Y.), 1.7 (M.I.), and 2.3 (M.H.). Thus, estimated $\sigma \mathrm{s}$ were all about 2.0. The results in Experiment 1 showed that $78 \%$ percent correct was obtained when the relative disparity between the outer planes was about 2-4 min of arc. These values are roughly in accordance with those of Stevenson et al. (1989). They assume a crosscorrelation function between two spread functions of the retinal images and estimate its width to be about $2 \mathrm{~min}$ of arc, which corresponds to the gap resolution acuity. However, according to the Reyrleigh criterion, it is necessary that the relative disparity reach at least about $2 \sigma$ in order to separate the overlapped pattern (Howard \& Rogers, 1995). Along this line, the estimated $\sigma \mathrm{s}$ in Experiment 3 were a bit larger than those in Experiment 1 (i.e., about $1-2$ min of arc). This is partly due to the property that we did not need to discriminate between falsely grouped dots and truly grouped ones with the global method but were faced with the problem of whether an arbitrary dot was grouped truly with the local method. Thus, a more distinct depth separation will be needed in the latter case. In addition, the task in Experiment 1 might be related largely to the averaging process, where the $p d f$ for averaged disparity will have smaller spreading. At any rate, we can say that these results suggest that the tasks in Experiments 1 and 3 reflect different processes involved in the depth separation. 


\section{GENERAL DISCUSSION}

In the present study, we examined the possibility that the depth separation of overlapped dot planes is enhanced by a symmetry structure. For this problem to be dealt with, we used two methods. In a direct and global method, we presented three or two overlapped dot planes consisting of symmetrical or random dots. The subjects were required to discriminate between three overlapped and two overlapped planes. In the other indirect and local method, we presented two overlapped dot planes and required the subjects to discriminate the depth positions of the planes from which the target dot (or dots) disappeared in the latter part of the stimulus presentation time. The results obtained in three experiments showed that the symmetry structure had no clear effects on the depth separation and that the discrimination performance improved with the relative disparity between dot planes, irrespective of whether the dot pattern had a symmetrical structure or not. These results indicate that a detection process for symmetry structure on a 2-D plane in 3-D space will not have a direct or an indirect connection (e.g., via a feedback loop) to a process involved in the depth separation. Recent research puts a stress on regularities in constructing internal representations of 3-D objects (Liu, Knill, \& Kersten, 1995; Poggio \& Vetter, 1992; Rock \& DiVita,1987). Our results suggest that the regularities are not made use of at the stage of depth separation of 3-D objects before the matching process of 3-D object recognition.

We used a new procedure in Experiments 2 and 3, and one problem for it might be pointed out: Whether the task in Experiments 2 and 3 really measured perceived depth separation of the dot planes. It is possible that our subjects adopted a strategy of responding to the disparity of the target, relative to the range of disparities of the other dots in the display. We thought that this possibility was slight. First, this is so because using missing dots as a target was not an appropriate strategy. If the subjects had paid attention to the target, they could have judged its depth position on the basis of its disparity, relative to the disparity range. However, in our task, the target was not defined at the start of a trial, and so the subjects should have paid attention to the dot planes, not to each dot. Second, the possibility seems slight because performance would have been improved had the subjects adopted that strategy. If we judge the depth position of the target on the basis of its relative disparity, the task will be related to the stereo-acuity and super-resolution tasks (Stevenson et al., 1989). The performance for those tasks was higher than that for the gap resolution task, which measured the depth separation of dot planes (Stevenson et al., 1989). The results in Experiments 2 and 3 showed that performance was lower than that in Experiment 1, where we directly measured the depth separation of dot planes.

On the basis of these accounts, we thought that our tasks in Experiments 2 and 3 depended on the depth sep- aration of two planes. However, it is possible that our subjects adopted that strategy and that, at the same time, our procedure of using missing dots as the target degraded their performance level. Thus, we should conduct further studies in order to resolve this problem

Our results can be partly described by a model considering two properties of the visual system: the distribution of internal representation of the binocular disparity and the masking effect of the off-attended surface. The term masking effect refers to the negative interaction induced by the process in which the image of components contained in one plane is projected on the other plane in the case that one surface is transparent. Because we assumed that the structure information would have potential effects on the former property, we shall focus on it. The optical and transmission subsystems in our visual system send the disparity information as a kind of probability variable. Thus, the discrimination performance will depend on the parameters, in particular the variance $\sigma^{2}$, of an internal probability function of the disparity. We assumed two processes, the grouping and averaging processes, both of which will have the Gaussianlike $p d f$ of the internal disparity. In this regard, there is one problem: the $p d f$ in the averaging process is not Gaussian when the grouping is completely done. In such a case, the sampling in the averaging process is restricted within the dots in each group of internal disparities of which the values have a lower or an upper limit. However, the perfect grouping would be a rare case. As to the averaging process, the $p d f$ in the process should have a smaller variance because the $p d f$ is on the sampled mean, and the variance will decrease with the size of the sample. In this regard, the size of the sample will be estimated by using efficiency analysis. Harris and Parker (1992) estimated the size of the sample for a depth discrimination task in which stimulus patterns consisted of random dots. We are now carrying out some experiments to estimate the size of the sample for the depth separation of overlapped dot planes by using efficiency analysis.

\section{REFERENCES}

Gescheider, G. A. (1985). Psychophysics: Method, theory, and application (2nd ed.). Hillsdale, $\mathrm{NJ}$ : Erlbaum.

Harris, J. M., \& PARKer, A. J. (1992). Efficiency of stereopsis in random-dot stereogram. Journal of the Optical Society of America A, 9, 1-12.

Howard, I. P., \& Rogers, B. J. (1995). Binocular vision and stereopsis. New York: Oxford University Press.

Liu, Z. , KNill, D. C. , \& Kersten, D. (1995). Object classification for human and ideal observers. Vision Research, 35, 549-568.

Nakayama, K., Shimojo, S., \& Ramachandran, V. S. (1990). Transparency: Relation to depth, subjective contours, luminance, and neon color spreading. Perception, 19, 497-513.

Parker, A. J., Johnston, E. B., Mansfield, J. S., \& YANG, Y. (1991) Stereo, surfaces, and shape. In M. S. Landy \& J. A. Movshon (Eds.), Computational models of visual processing (pp. 359-381). Cambridge, MA: MIT Press.

PARKer, A. J., \& YANG, Y. (1989). Spatial properties of disparity pooling in human stereo vision. Vision Research, 29, 1525-1538.

POGgIO, T., \& VETTER, T. (1992). Recognition and structure from one 
$2 D$ model view: Observations on prototypes, object classes and symmetries (A.I. Memo No. 1347). Cambridge, MA: MIT, Artificial Intelligence Laboratory.

Rock, I., \& DiVITA, J. (1987). A case of viewer-centered object perception. Cognitive Psychology, 19, 280-293.

Stevenson, S. B., Cormack, L. K., \& Schor, C. M. (1989). Hyperacuity, superresolution and gap resolution in human stereopsis. $\mathrm{Vi}$ sion Research, 29, 1597-1605.

TYler, C. W. (1983) Sensory processing of binocular disparity. In C. M. Schor, K. Ciuffereda, \& M. Woburn (Eds.), Vergence eye movements: Basic and clinical aspects (pp. 99-296). Boston: Butterworth.
WiNer, B. J. (1962). Statistical principles in experimental design. New York: McGraw-Hill.

YaKuShiJin, R., \& IsHiguchi, A. (1999). The effect of a noise plane on discrimination of mirror-symmetry in a different depth plane. Journal of Experimental Psychology: Human Perception \& Performance, 25, $1-12$.

(Manuscript received April 15, 1997; revision accepted for publication December $31,1997$. 